\title{
Zinc: The Wonder Drug for the Treatment of Carcinomas
}

\section{Leslie C Costello* and Renty B Franklin}

Department of Oncology and the Greenebaum Comprehensive Cancer Center, The University of Maryland School of Dentistry, Baltimore, Maryland, USA

*Corresponding Author: Leslie C Costello, Department of Oncology and Diagnostic Sciences, The University of Maryland, Baltimore, Maryland, USA.
Received: March 03, 2020

Published: April 27, 2020

(C) All rights are reserved by Leslie $\mathbf{C}$ Costello and Renty B Franklin .

\section{Abstract}

Evidence is evolving that support the relationship that all carcinomas exhibit the following important relationships: The malignant cells exhibit a significant decreased zinc compared to the normal cells. The higher zinc levels that exist in the normal cells are cytotoxic in the malignant cells. The decrease in zinc is due to the down regulation of the ZIP-family zinc uptake transporter. These cells are as "ZIP-deficient/decreased zinc" malignancies. This provides a target for a chemotherapy that can restore the high zinc levels that will manifest cytotoxic effects in the malignant cells. In order to achieve this, a vehicle that facilitates the uptake and accumulation of zinc in the ZIP-deficient cells is required. The zinc ionophore, clioquinol, exhibits the properties that will provide these requirements. This is demonstrated by the treatment of a patient with $3 \%$ Clioquinol Cream, which successfully suppressed the progression of androgen-dependent prostate cancer. This treatment should also be efficacious for pancreatic cancer, liver cancer, breast cancer, thyroid cancer, kidney cancer, stomach cancer, gall bladder cancer, and lung cancer; which are carcinomas that exhibit decreased zinc. Thus, it is appropriate to describe that "Zinc is the wonder drug for the treatment of carcinomas".

Keywords: Zinc; Carcinomas; Clioquinol; Treatment

\section{Introduction}

In the 1993 hallmark review [1] of the physiological and pathophysiological implications of zinc, Vallee and Falchuk state: "In fact, zinc is the only pre-, post-, and transitional element that has proven to be essentially nontoxic...It is neither carcinogenic, mutagenic, nor teratogenic". This is represented by the treatment of Wilson's disease with $600 \mathrm{mg}$ zinc acetate/day. Wilson's disease is incurable; and most cases require the sustained treatment for the life of the patient. There is no evidence that this exposure to zinc promotes the development of cancer.

Many contemporary biomedical investigators and clinicians do not recognize, and/or accept, the important implications of zinc in the development, progression, and treatment of carcinomas (reviewed in [2]). It is in the interest of the medical community and the public welfare that the correct zinc relationships are established.

The status of zinc in blood plasma that is available for cell uptake: The normal blood plasma concentration of zinc is $12-15 \mathrm{uM}$. Virtually all of the zinc is bound to either high molecular weight proteins (e.g. globulin), or to low molecular weight solutes (such as albumin, amino acids, citric acid). The latter group comprises the interstitial fluid concentration of 2 - $4 \mathrm{uM}$ zinc, which constitutes "mobile Zn Ligands". This is the source of zinc that is transported into the cells. Notably, the concentration of free $\mathrm{Zn}^{++}$ion estimated is negligible (0.1 - $1.0 \mathrm{nM}$ ). Despite this, the vast majority of published reports mistakenly employ and/or refer to the zinc in plasma and other extracellular fluids as being "free zinc" or "free zinc ions" [3].
Zinc concentrations in specific carcinomas: The zinc concentrations in cancer versus normal tissue preparations are available for some cancers. However, the only fully reliable methodology must incorporate histochemical zinc analysis of tissue sections with an appropriate specific zinc staining, in order to establish the localization and relative concentration of zinc in the cells. Unfortunately, the majority of reports have employed the measurements of zinc concentrations in tissue extract preparations; which cannot differentiate the localization of zinc in the malignant cells compared to normal cells and other component of the tissue. That information is inconclusive and often misleading. The following description will focus on the cancers for which histochemical/histological verification was employed.

Zinc in prostate cancer: The most studied cancer zinc relationships is prostate cancer [4]. A reason is that the prostate gland has the unique specialized function of accumulating and secreting very high zinc levels. Table 1 shows the 10 -fold greater zinc concentration in the normal prostate peripheral zone compared to other soft tissues. Most importantly, the malignant peripheral zone (the main region where malignancy develops) exhibits a major decreased zinc compared to the normal peripheral zone and other soft tissues.

Figure 1 reveals, without exception, that zinc is significantly decreased in prostate cancer compared to normal prostate. In situ histochemical staining (Figure 2) reveals that most zinc is localized in the normal acinar secretory epithelial cells; and the stroma contains much lower zinc. In contrast, the malignant cells have minimal detectable zinc along with the stroma. Figure 3 shows the energy dispersion x-ray fluorescent zinc analysis of prostate tissue samples [5]. The malignant samples exhibited 70\% decreased zinc. 


\begin{tabular}{|c|c|}
\hline ( $\mu$ g/g wet weight) & Zinc \\
\hline Normal peripheral zone & 295 \\
\hline PCa peripheral zone & $30-50$ \\
\hline Other soft tissue & 30 \\
\hline Blood plasma & 1 \\
\hline
\end{tabular}

Table 1: Typical comparative prostate zinc levels.

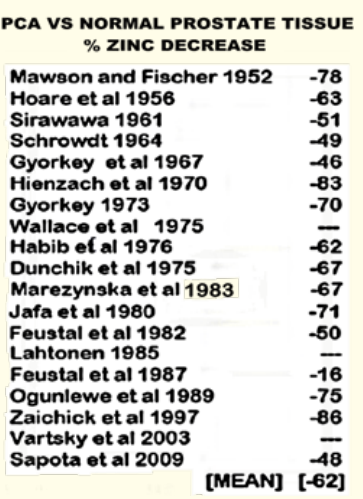

Figure 1: List of population reports reveals that all studies have demonstrated a significant decreased zinc in prostate cancer.

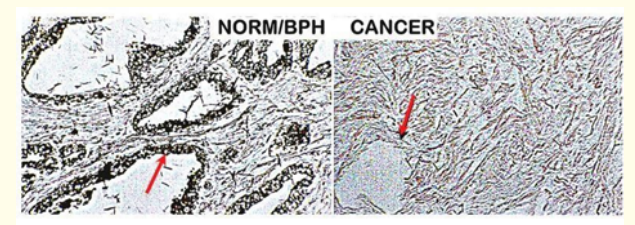

Figure 2: Dithizone stain of prostate tissue sections showing high zinc in normal/BPH acinar cells vs. loss of zinc in malignant cells.

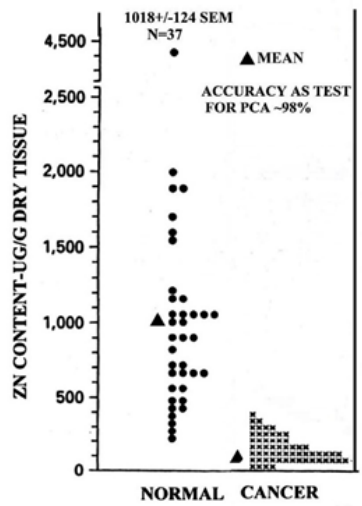

Figure 3: Energy dispersive x-ray fluorescent measurements of zinc in normal and cancer prostate tissue samples (modified from [5]).

Compared to the wide variation in normal prostate tissue zinc levels, cancer tissue zinc levels show extremely low variation. No cancer patient tissue exhibited a zinc concentration that approached the higher mean concentration of the normal prostate tissue.
Several hundred patients that are represented in these reports; which represent different stages of prostate cancer; different treatments; different ages; different diets and eating habits, and many other differences in life style and activities. Despite these extensive variations, the results are extremely consistent. The most significant relationship is that no reported prostate cancer case exists in which the zinc level of the malignant tissue is not decreased from that in the normal tissue. This is conclusive evidence that the higher levels of zinc in the normal cells are cytotoxic in the malignant cells.

Zinc in liver cancer (hepatic cell carcinoma): Beginning with Danielsen and Steinnis in 1970 [6], 10 reported population studies have consistently demonstrated a marked decrease (55 - 75\%) in zinc levels in HCC tissue compared with normal/benign liver tissue. Figure 4 shows the decreased zinc in the hepatoma cells compared to the normal hepatocytes. The consistency is represented in Kew and Mallet [7] (Figure 5), who showed that the zinc concentration in liver cancer tissue is significantly less than the normal cirrhotic or non-cirrhotic tissue $(\mathrm{P}<0.001)$. Similarly, Tashiro., et al. [8] reported that the zinc concentration in cancerous liver was lower (55\% decrease) than in all noncancerous liver tissue for all twenty-three cancer subjects. Our in situ histochemical analysis of zinc levels in tissue section samples [9] demonstrated that all 26 HCC cases exhibited major decreased zinc in the hepatoma cells in well-differentiated and advancing malignancy, compared to higher zinc in the normal hepatocytes (Figure 4).

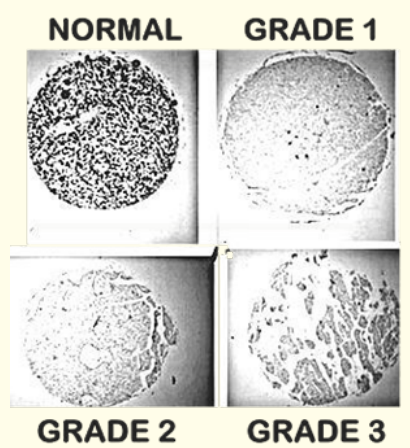

Figure 4: Dithizone stain of liver tissue sections shows marked decreased zinc in malignancy.

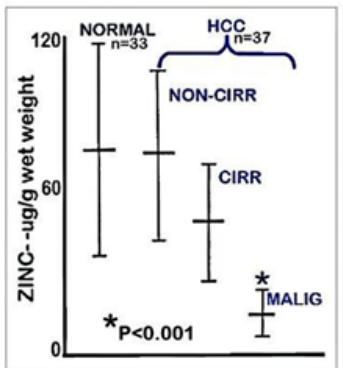

Figure 5: Liver zinc levels show that zinc is always decreased in malignancy.

Zinc in pancreatic cancer: Aside from our studies [10], no other reports exists that provide the determination of zinc levels in normal and cancer tissues. The in situ histochemical staining of pancreatic tissue sections revealed that all grades of the 64 can- 
cer cases exhibited major decreased zinc when compared with the normal pancreas (Figure 6).

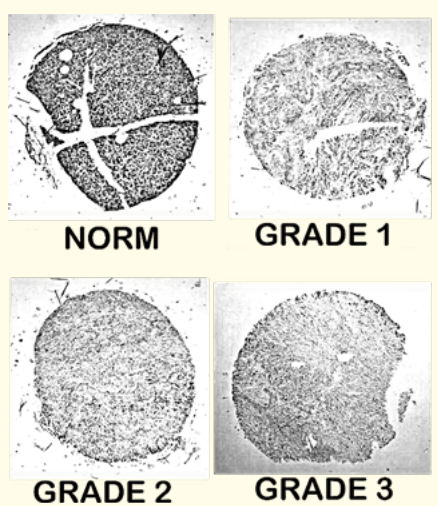

Figure 6: Dithizone stain of pancreatic tissue sections shows marked decreased zinc in malignancy.

Zinc in breast cancer: There exist seven reports that consistently purport to demonstrate with breast tissue extract preparations that the zinc levels are increased in breast ductal cancer. If correct, breast cancer would be the only carcinoma in which zinc promotes the progression of malignancy. More- than other tissues, the histology of bre ast includes the complexity of different coexisting tissues; which imposes a greater necessity for in situ histochemical zinc analysis to identify the localization of zinc levels.

Figure 7A shows that the malignant ducts exhibit major decreased zinc compared to the normal ductal epithelium. This persistent zinc decrease exists in all stages of malignancy (Figure 7C). Figure 7B reveals that the adipose tissue does not exhibit detectable zinc [11]. Conversely, the existence of necrotic tissue exhibits high zinc levels. Therefore, breast tissue preparations or extracts cannot be employed for the specific determination of zinc levels in malignant versus normal ducts.

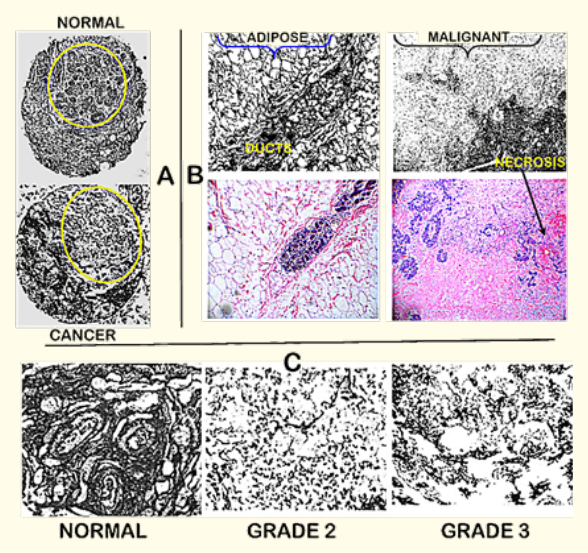

Figure 7: Zinc staining and histology to show the localization of zinc in breast tissue components.

Consequently, the reports of increased zinc in breast cancer, do not represent the status of zinc in the normal versus malignant ductal epithelium. Notably, those reports also identify simultaneous changes in 11 other elements. Such results are not likely to exist, or be relevant, in a malignant cell. Seemingly, this conundrum should have dictated the requirement for histological and histochemical verification of zinc, as we have done.

Why do carcinomas exhibit significantly decreased zinc compared to their corresponding normal cells?

There is no corroborated case report of prostate, liver, or pancreatic cancer in which the malignant cells retain the higher zinc concentration that exists in the normal cell. The reason is that the normal cell zinc levels are cytotoxic in the malignant cells. Therefore, the malignant cells evolved with mechanisms that prevent the accumulation of the cytotoxic higher zinc levels.

The downregulation of ZIP-family zinc uptake transporters (SIc39A) in malignant cells

All mammalian cells contain a ZIP-family zinc-uptake transporter that is localize in the plasma membrane [4]. In the malignant cells, the ZIP transporter is downregulated: i.e. malignant cells are "ZIP-deficient" cells; which limits the uptake and accumulation of zinc. This is revealed with in situ immunohistochemistry of tissue sections; which demonstrates that plasma membrane localized ZIP1, ZIP3, and ZIP14 transporters exist in normal prostate, pancreas, and liver, respectively (Figure 8). Notably, detectable localized plasma membrane ZIP transporters do not exist in the malignant cells.

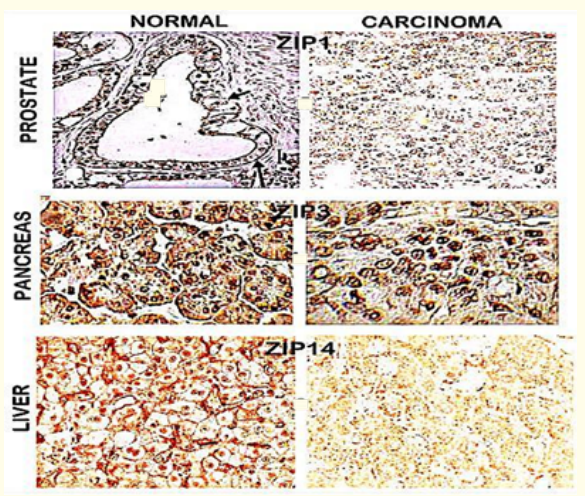

Figure 8: In situ immunohistochemistry of ZIP transporters in prostate, pancreas, and liver tissue sections.

The zinc ionophore approach as a chemotherapy to terminate carcinomas

The preceding relationships make it apparent that a zinc chemotherapy requires an agent that will facilitate the transport of cytotoxic levels of zinc into ZIP-deficient carcinomas. The zinc ionophore, clioquinol (5-chloro-7-iodo-8-quinolinol), was elected because of its ideal zinc-binding properties. Clioquinol exhibits a zinc binding formation constant $\log \mathrm{Kf}=7$ - 8, which binds blood plasma zinc as a mobile, reactive zinc ligand ( $\mathrm{ZnCQ})$; and when transported into the malignant cell, the zinc is released and manifests its cytotoxic effects. This is confirmed with experimental studies [12], in which clioquinol treatment resulted in $85 \%$ inhibition of human prostate tumor growth in the nude mice xenograft model (Figure 9). 


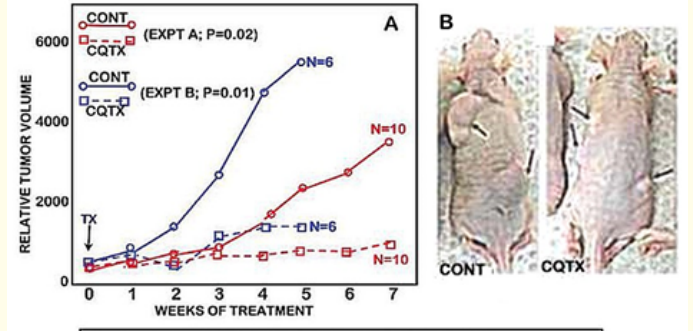

C EFFECT OF CQTX ON TOTAL TUMOR GROWTH

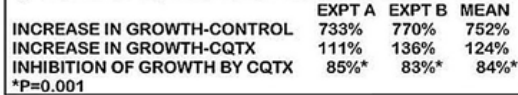

Figure 9: Effects of two experiments of clioquinol treatment of human prostate tumor growth in xenograft mice.

The hormonal types and development of advanced prostate cancers

There exists two types of advanced prostate cancer: "androgendependent" and "androgen-independent" prostate cancers. The latter is referred to as castration (or hormone)-resistant prostate cancer (CRPC), for which its manifestation was unknown. The prevailing concept has been that androgen-ablation attenuates, but does not terminate, the prostate malignancy. Then, when plasma testosterone is not available, the malignancy persists as "androgen-independent" malignancy. However, the manifestation of the development and progression of the "androgen-independent" malignancy remained unknown.

Those relationships and prevailing view are no longer tenable. The evolving evidence $[13,14]$ is now supportive of the new understanding that "androgen-independent" malignancy is manifested by prolactin; i.e. "prolactin-dependent" prostate cancer. Either prostate cancer can exist in patients; or both prostate cancers can simultaneously co-exist in patients.

Then the issue is why these two different and separate prostate cancers were not known to exist. The major reason is that "androgen-dependent" malignant cells can be identified by their expression and production of biomarkers such as increased PSA (Prostate Specific Antigen) and PSMA (Prostate Specific Membrane Antigen). Those biomarkers do not exist for "prolactin-dependent" malignancy. Consequently, PSA level and PSMA PET/CT for identification or treatment of malignancy can misrepresent the status of malignancy if "prolactin-dependent" malignancy exists. Negative results will be interpreted as the absence of detectable malignancy; whereas the patient could be facing imminent death from advanced prolactin-dependent prostate cancer, generally in 5 years or less.

The prevailing concept of the development and progression of prostate malignancy also requires reconsideration. The initiation of the malignant process results from conditions that activate the oncogenic factors that transform the normal acinar epithelial cell to a premalignant cell, which has malignancy potential. The premalignant cell undergoes "genetic/metabolic" transformation, which provides the energetic and synthetic requirements for the malignant activities of the developing malignant cells. The initiating oncogenic event is not under hormonal regulation. Hormonal influence occurs in promoting the development and progression of the malignant cell.

The normal prostate acinar epithelial cell contains the receptors and signaling pathways for testosterone and prolactin. Therefore, the initiation and early development of prostate cancer can be promoted by either testosterone and by prolactin; and lead to "androgen-dependent" advanced prostate cancer; and "prolactindependent" advanced prostate cancer. The prevailing view has been based on the concept of Dr. Huggins (Nobel Laureate) that the castration/hormonal ablation treatment for androgen-dependent prostate cancer leads to the development of androgen-independent prostate cancer. That view must be abandoned and replaced by the appropriate understanding that prolactin-manifested prostate cancer exists in some of the patients with androgen-dependent prostate cancer. This is why Dr. Huggins correctly noted that some, but not all, patients exhibited this status of HRPC prostate cancer.

Chemotherapies for the treatment of terminal advanced prostate cancer: In recent years, about 230,000 new cases/year; and about 30,000 deaths/year due to prostate cancer occur in the USA. About 1.1 million cases/year and 307,000 deaths/year occur worldwide. Most of these deaths are due to a terminal advanced prostate cancer, for which an established chemotherapy has not existed.

Over time, we examined many slides of normal prostate tissue and prostate malignant tissue; including tissue microarrays containing cases of normal and varying stages of malignancy. Zinc staining consistently revealed the prominent zinc staining of the normal acinar epithelium and the absence of detectable zinc in malignancy. The staining does not differentiate "androgen-dependent" malignancy from "prolactin-dependent" malignancy; and it would be expected that either one or both is represented in some of the cases. Based on these relationships, an efficacious chemotherapy must be considered in relation to the prostate malignancy that exists in the patient.

The case report of the first successful zinc treatment for terminal advanced prostate cancer: A recent case report [15] represents the relationships described above. The patient had received the typical androgen ablation therapy. When treatment with $3 \%$ Clioquinol Cream plus $50 \mathrm{mg}$ zinc supplement/day was initiated, the androgen-independent malignancy was suppressed. This was due to the increased accumulation of zinc and its cytotoxic effects on the malignant cells.

The existence of prolactin-dependent malignancy was revealed. Cabergoline treatment (dopamine agonist) was initiated to inhibit the pituitary production of prolactin. The plasma prolactin decreased by $88 \%$; and aborted the prolactin-dependent malignancy. This combination treatment regimen is the first case report of a successful treatment of a patient with terminal advanced prostate cancer. 
The potential of zinc treatment for liver and pancreatic carcinomas: Pancreatic cancer (adenocarcinoma) accounts for about 44,000 new cases/year and 38,000 death/year in the U.S. will occur [16,17]. Liver cancer accounts for about 28,000 cases, of which $\sim 75 \%$ results in death. Both cancers have 5-year survival rates of about 6-years; and about $90 \%$ death will occur within the first 2 years. Moreover, there has been little improvement in these survival rates over the past 30 years; and liver cancer incidence continues to increase.

This lack of significant progress in dealing with these cancers is largely due to the poor understanding and resolution of the factors and events involved in the development of the malignancy and its progression to advanced stages. The consequence has been the absence of an efficacious chemotherapy that will terminate these malignancies.

Unfortunately, most clinicians and biomedical investigators are not aware, or chose to ignore the implications of zinc in pancreatic and liver cancers. Both cancers exhibit the same zinc and ZIP transporter relationships as exists in prostate cancer. Figure 10 shows that, unlike the in situ pancreatic cancer status, the Panc1 cell line exhibits the expression of ZIP3; and zinc treatment results in increased uptake of zinc that inhibits cell proliferation. Similarly, the liver HepG2 cells exhibit ZIP14 plasma membrane-localized transporter; and increased uptake of zinc results in the inhibition of cell proliferation (Figure 11).

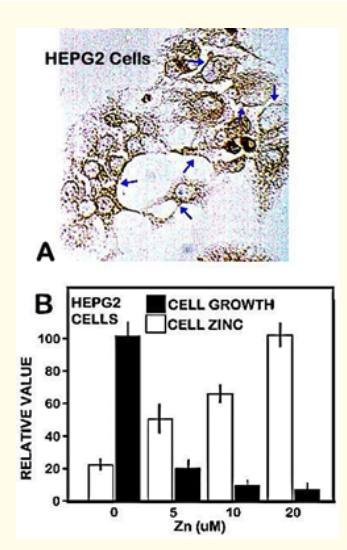

Figure 10: A. ZIP3 transporter localized at the plasma membrane in HepG2 liver cells. B. The uptake of zinc and its effect on cell growth.

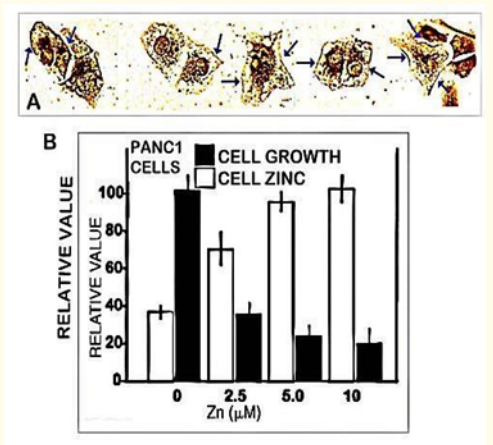

Figure 11: A. ZIP3 plasma membrane localization in Panc1 cells. B. The uptake of zinc and its effect on cell growth.
The status of zinc in other carcinomas: As described above (Figure 7), breast cancer has also been shown to exhibit decreased zinc. For other tissues, no reports exist that employed appropriate zinc staining to determine the in situ status of zinc in human tissue sections. There are reports that kidney, thyroid, stomach, lung, and gall bladder normal and cancer tissue extracts preparations exhibit decreased zinc compared to the corresponding normal tissue extracts [18]. Those reports need to be corroborated by in situ zinc staining of tissue sections. Nevertheless, the available studies provide compelling evidence for the conclusion that "all carcinomas exhibit decreased zinc". This underscores the relationship that "The higher level of zinc that exists in the normal cells is cytotoxic in the malignant cells". It will be relevant to establish that the respective ZIP transporter is the prevalent factor for this zinc status.

The cytotoxic effects of zinc in malignant cells: In 1999, Liang., et al. [19] first reported that "zinc inhibits human prostatic carcinoma cell growth, possibly due to induction of cell cycle arrest and apoptosis". Zinc promotion of mitochondrial apoptogenesis represents the most common and direct cytotoxic mechanism for prostate malignancy. Zinc induces the expression of Bax and its incorporation into the mitochondrial membrane; which is followed by the release of cytochrome c, and its induction of caspase-promoted apoptosis (Figure 12) [20].

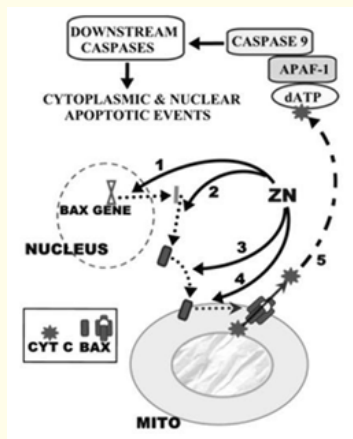

Figure 12: Zinc promotion of mitochondrial apoptogenesis. 1-4 shows $\mathrm{Zn}$ recruitment of Bax to mitochondrial membrane for release of cytochrome $c$.

In addition, the cytotoxic effects of zinc include: the inhibition of mobility and invasion of malignant cells; and changes in intermediary metabolism and terminal electron transport in order to provide the bioenergetic and synthetic requirements for malignancy [21].

The misrepresented view of epidemiology reports that zinc treatment promotes prostate cancer; and its impact: Despite the above-established relationships, some epidemiology reports have and continue to promote the erroneous view that men who take zinc supplements greater than the normal daily requirement are at greater risk for promoting the progression of prostate cancer (reviewed in [22]). The most notable is the 2003 Leitzman., et al. epidemiology report [23], which concluded that: "Excessively high supplemental zinc intake was associated with an increased risk of advanced prostate cancer". Because the report emanated from NIH and Harvard University, it resulted in highly publicized announcements, such as: HealthDay News (July 2, 2003), "Men who over- 
dose on zinc supplements more than double their risk of prostate cancer, a government study finds"; The Washington Post (July 1, 2003), "Study Links Zinc, Prostate Cancer. Men who take too much zinc may be raising their prostate cancer risk, U.S. researchers said yesterday"; The Mayo Clinic Health Letter (May 2004) "Large doses of zinc may increase risk of prostate cancer".

This was the dominant message to the medical community and the general public; despite the existence of other epidemiology reports that have suggested zinc supplement protects against prostate $[22,24]$. In fact, the Leitzmann., et al. report had also revealed that moderate zinc supplement protected against the progression of prostate cancer; which they did not recognize. Notably, the 2011 Epstein et al epidemiology report [25] suggested that "high dietary intake of zinc is associated with lower prostate cancer-specific mortality after diagnosis, particularly in men with localized disease"; and one of the authors was co-author on the Leitzmann., et al. report; thereby reversing the earlier conclusion. The 2016 epidemiology report of Mahmoud., et al. [26] also suggested a protective effect of zinc intake. Yet, the Leitzman., et al. report has continued to dominate the prevailing views of many (possibly most) of the contemporary medical community and the general public.

That is illustrative of the admonitions expressed by other highly respected epidemiologists [24,27]. Dr. Dimitrios Trichopoulos stated “...epidemiology studies will inevitably generate false positive and false negative results with disturbing frequency... we may do more harm than good". Dr. Brian MacMahon, "...the tentative suggestion of an association is likely to be interpreted as a fact". Dr. Alex Walker stated, "...once a possible link is in the public eye, it can be virtually impossible to discredit". These admonitions are applicable to the concerns that we have raised regarding unsubstantiated and questionable epidemiology reports that purport to establish an effect of dietary/supplemental zinc and promotion of prostate cancer.

\section{Conclusion}

1. All carcinomas are zinc-deficient malignancies. The higher zinc levels that exist in the normal cells are cytotoxic in the malignant cells.

2. The decrease in zinc is due to the down regulation of the ZIPfamily zinc uptake transporter. Therefore, the carcinomas are characterized as "ZIP-deficient/decreased zinc" malignancies.

3. The restoration of high zinc levels in the malignant cells is a target for a zinc chemotherapy. This requires a vehicle to facilitate the uptake and accumulation of cytotoxic levels of zinc in the ZIP-deficient cells.

4. The zinc ionophore, clioquinol, exhibits the properties that will achieve the above requirements. The treatment of a patient with 3\% Clioquinol Cream successfully suppressed the progression of androgen-dependent prostate cancer.

5. Clioquinol treatment should also be efficacious for pancreatic cancer, liver cancer, breast cancer, thyroid cancer, kidney cancer, stomach cancer, pulmonary carcinoma, and gall bladder cancer; all exhibiting decreased zinc.
6. It is reasonable to conclude that "all carcinomas exhibit decreased zinc"; and that the zinc ionophore, clioquinol, will be an efficacious chemotherapy for these carcinomas".

7. Therefore, it is well founded to pronounce that: "Zinc is the Wonder Drug for the Treatment of Carcinomas".

\section{Bibliography}

1. Vallee B and Falchuck K. "The biochemical basis of zinc physiology". Physiological Reviews 73 (1993): 79-118.

2. Costello LC and Franklin RB. "A comprehensive review of the role of zinc in normal prostate function and metabolism; and its implications in prostate cancer". Archives of Biochemistry and Biophysics 611 (2016):100-112.

3. Costello LC., et al. "Evidence for operation of the direct zinc ligand exchange mechanism for trafficking, transport, and reactivity of zinc in mammalian cells". Journal of Inorganic Biochemistry 105 (2011): 589-599.

4. Franklin RB., et al. "Zinc and zinc transporters in normal prostate and the pathogenesis of prostate cancer". Frontiers in Bioscience 10 (2005): 2230-2239.

5. Zaichick VY., et al. "Zinc in human prostate gland: Normal, hyperplastic and cancerous". Journal of Radioanalytical and Nuclear Chemistry 217 (1997): 157-161.

6. Danielsen A and Steinnes E. "A study of some selected trace elements in normal and cancerous tissue by neutron activation analysis". The Journal of Nuclear Medicine 11 (1970): 2602626034.

7. Kew MC and Mallett RC. "Hepatic zinc concentrations in primary cancer of the liver". British Journal of Cancer 9 (1974): 80-83.

8. Tashiro H., et al. "Variation in the distribution of trace elements in hepatoma". Biological Trace Element Research 95 (2003): 49-63.

9. Costello LC and Franklin RB. "The status of zinc in the development of hepatocellular cancer: an important, but neglected, clinically established relationship". Cancer Biology and Therapy 15 (2014): 353-360.

10. Costello LC., et al. "Decreased zinc and downregulation of ZIP3 zinc uptake transporter in the development of pancreatic adenocarcinoma". Cancer Biology and Therapy 12 (2011): 297 303.

11. Costello LC., et al. "In situ clinical evidence that zinc levels are decreased in breast invasive ductal carcinoma". Cancer Causes and Control 27 (2016): 729-735.

12. Costello LC., et al. "Evidence that human prostate cancer is a ZIP1-Deficient malignancy that could be effectively treated with a zinc Ionophore (clioquinol) approach". Chemotherapy 4 (2015): 15213. 
13. Costello LC. "The suppression of prolactin is required for the treatment of advanced prostate cancer". Oncogene 2.3 (2019): 13.

14. Costello LC and Franklin RB. "Testosterone, prolactin, and oncogenic regulation of the prostate gland. A new concept: Testosterone-independent malignancy is the development of prolactin-dependent malignancy!". Oncology Reviews 12 (2018): 356.

15. Costello LC., et al. "A novel patient case report to show the successful termination of untreatable androgen-independent prostate cancer: Treatment with cabergoline (dopamine agonist)”. Mathews Open Access Journals 4.1 (2019): 42.

16. Costello LC and Franklin RB. "A review of the current status and concept of the emerging Implications of zinc and zinc transporters in the development of pancreatic Cancer". Pancreatic Disorders and Therapy 4 (2013): 002.

17. Siegel RL., et al. "Cancer statistics, 2019”. CA: A Cancer Journal for Clinicians 69 (2019): 7-34.

18. Gumulec J., et al. "Serum and tissue zinc in epithelial malignancies: a meta-analysis". PLoS One 9 (2014): e99790.

19. Liang JY., et al. "Inhibitory effect of zinc on human prostatic carcinoma cell growth". Prostate 40 (1999): 200-207.

20. Feng P., et al. "Direct effect of zinc on mitochondrial apoptogenesis in prostate cells". Prostate 52 (2002): 311-318.

21. Franklin RB and Costello LC. "Zinc as an anti-tumor agent in prostate cancer and in other cancers". Archives of Biochemistry and Biophysics 463 (2007): 211-217.

22. Costello LC Franklin RB., et al. "Zinc and prostate cancer: a critical scientific, medical, and public interest issue (United States)". Cancer Causes and Control 16 (2005): 901-915.

23. Leitzmann MF., et al. "Zinc supplement use and risk of prostate cancer". Journal of the National Cancer Institute 95 (2003): 1004-1007.

24. Costello LC., et al. "A critical assessment of epidemiology studies regarding dietary/supplemental zinc and prostate cancer risk". The Open Urology and Nephrology Journal 1 (2008): 10.

25. Epstein MM., et al. "Dietary zinc and prostate cancer survival in a Swedish cohort". The American Journal of Clinical Nutrition 93 (2011): 586-593.

26. Mahmoud AM., et al. "Zinc Intake and Risk of Prostate Cancer: Case-Control Study and Meta-Analysis". PLoS One 8 (2016): 11.

27. Taubes G. "Epidemiology faces its limits". Science 269 (1995): 164-169.

\section{Assets from publication with us}

- Prompt Acknowledgement after receiving the article

- Thorough Double blinded peer review

- Rapid Publication

- Issue of Publication Certificate

- High visibility of your Published work

Website: https://www.actascientific.com/

Submit Article: https://www.actascientific.com/submission.php Email us: editor@actascientific.com

Contact us: +919182824667 